\title{
TI.104.1
}

\section{InCommon Technical Advisory Committee Charter}

- PDF: InCommonTechnicalAdvisoryCommitteeCharter.pdf

- Text: InCommonTechnicalAdvisoryCommitteeCharter.txt

\section{More Information}

\begin{tabular}{|c|c|}
\hline Repository ID & TI.104.1 \\
\hline Persistent URL & http://doi.org/10.26869/TI.104.1 \\
\hline Title & InCommon Technical Advisory Committee Charter \\
\hline Authors & InCommon Technical Advisory Committee \\
\hline Sponsor & InCommon Steering Committee \\
\hline \multicolumn{2}{|l|}{ Review } \\
\hline Status & Legacy \\
\hline Publish Date & December 7, 2015 \\
\hline DOI & 10.26869/TI.104.1 \\
\hline \multicolumn{2}{|l|}{ Signature } \\
\hline Deprecated & No \\
\hline \multicolumn{2}{|l|}{ Future Review } \\
\hline \multicolumn{2}{|l|}{ Supersedes } \\
\hline Format & PDF, Text \\
\hline \multicolumn{2}{|l|}{ Related Docs } \\
\hline \multicolumn{2}{|l|}{ Development Location } \\
\hline \multicolumn{2}{|l|}{ IP Framework } \\
\hline Subject Tags & federation, incommon \\
\hline Notes & As of 2018 , the AAC was replaced by the CTAB. See the CTAB charter here http://doi.org/10.26869/TI.94.1 \\
\hline
\end{tabular}

